FORMATION Formation emploi

Revue française de sciences sociales

$110 \mid 2010$

Pêle-mêle

\title{
L'enseignement supérieur en France : un espace segmenté qui limite l'égalisation des chances
}

Higher education in france: a segmented space that limits equal opportunity

Das höhere bildungswesen in frankreich : ein segmentierte markt, der der

chancengleichheit grenzen setzt

La enseñanza superior en Francia: un espacio segmentado que limita la igualdad de oportunidades

\section{Elise Verley et Sandra Zilloniz}

\section{OpenEdition}

Journals

Édition électronique

URL : http://journals.openedition.org/formationemploi/2914

ISSN : 2107-0946

Éditeur

La Documentation française

Édition imprimée

Date de publication : 15 juin 2010

Pagination : 5-18

ISSN : 0759-6340

Référence électronique

Elise Verley et Sandra Zilloniz, "L'enseignement supérieur en France : un espace segmenté qui limite l'égalisation des chances », Formation emploi [En ligne], 110 | 2010, mis en ligne le 10 septembre 2012, consulté le 30 octobre 2020. URL : http://journals.openedition.org/formationemploi/2914

(C) Tous droits réservés 


\title{
Formation
}

\section{L'enseignement supérieur en France: un espace segmenté qui limite l'égalisation des chances}

Par Elise Verley et Sandra Zilloniz*

\begin{abstract}
Les différences entre étudiants s'organisent selon le secteur d'études : sélectif, professionnalisant ou universitaire. Des oppositions existent entre ces secteurs en termes de parcours, comportements et caractéristiques des étudiants.
\end{abstract}

La massification scolaire, caractérisée par une croissance du nombre de collégiens, de lycéens, d'étudiants mais aussi de diplômes, a transformé le paysage de l'enseignement supérieur. Les effectifs universitaires ont connu une croissance continue depuis les années $60^{1}$, alors que leur poids relatif au sein de l'ensemble de l'enseignement supérieur a eu tendance à diminuer, soit presque un étudiant du supérieur sur deux fréquentant l'université 2 . Cette décroissance relative n'est pas due à une baisse du nombre d'inscrits dans les universités ${ }^{3}$, mais au développement beaucoup plus marqué d'autres établissements d'enseignement supérieur (Galland et Oberti, 1996), en réponse à une demande accrue de formations professionnalisantes.

\footnotetext{
${ }^{1}$ Bien qu'à partir des années 2000, on assiste à une légère baisse des effectifs, qui correspond à un tassement démographique mais aussi à une baisse des taux d'obtention du baccalauréat général. Parallèlement, depuis la fin des années 90, l'allongement des études paraît bel et bien stoppé.

${ }^{2} 56 \%$ hors IUT (instituts universitaires de technologie) en 2007 contre $68 \%$ trente ans plus tôt.

${ }^{3}$ Les flux d'entrée à l'université diminuent à partir de 1995-1996 et passent de 278447 à 239574 en 2005-2006.
}

* Elise Verley est sociologue, maître de conférences en sociologie à l'université Paris Sorbonne - Paris IV, membre du GEMASS IGroupe d'études et de méthodes de l'analyse sociologique de la Sorbonne) et chargée de mission à l'Observatoire national de la vie étudiante. Ses domaines d'investigations sont la sociologie de l'emploi, de l'éducation, des publics universitaires. Elle a notamment publié, avec Marie Duru-Bellat, " Les étudiants au fil du temps : contexte et repères institutionnels ", in Les étudiants en France. Histoire et sociologie d'une nouvelle jeunesse, PUR, novembre 2009

Sandra Zilloniz est statisticienne, ingénieur d'études à l'Observatoire national de la vie étudiante. Ses domaines d'investigation ont trait aux conditions de vie et d'études des publics de l'enseignement supérieur. Elle a publié récemment : "La gestion du corps : alimentation et santé ", in Les étudiants en France. Histoire et sociologie d'une nouvelle jeunesse, PUR, novembre 2009 et avec Ronan Vourc'h, "Indicators for France ", in Schnitzer K. (Dir.), Eurostudent Report, Social and Economic Conditions of Student Life, Hanovre, Synopsis of indicators, HIS, 2008. 
C'est le cas en particulier des sections de techniciens supérieurs (STS) et des instituts universitaires de technologie (IUT) qui, sur la longue période, ont vu leurs effectifs augmenter de façon considérable jusqu'aux années 2000, et, dans une moindre mesure, des classes préparatoires aux grandes écoles (CPGE). Ces évolutions quantitatives doivent donc être rapportées à un mouvement de fond lié à la diversification et à la professionnalisation des cursus de l'enseignement supérieur.

La diversification des structures, que l'on peut considérer comme une adaptation à ces évolutions et une condition de l'expansion (Duru-Bellat et Verley, 2009), a conduit à une fragmentation de l'expérience étudiante et à une segmentation de l'enseignement supérieur désignant la « subdivision des systèmes d'enseignements en écoles et programmes parallèles se distinguant à la fois par les cursus, l'origine sociale de leurs élèves, la réputation et le prestige » (Ringer, 2003). C'est ce qu'atteste, dans le système français, l'existence de deux secteurs d'enseignement qui s'opposent par leur sélectivité à l'entrée : un secteur dit «fermé », parce que plus ou moins sélectif, dans lequel les étudiants sont recrutés sur dossier ou concours d'entrée, et un secteur dit « ouvert », correspondant aux universités. Dans le secteur fermé, on distingue les études courtes professionnalisantes (STS, IUT) des classes préparatoires aux grandes écoles qui destinent à des parcours spécialisés et élitistes. Dans le secteur « ouvert », les études universitaires de Santé (en particulier médecine) se distinguent des autres filières de l'université, non sélectives, par un recrutement sur concours à la fin de la première année d'études.

Pour permettre la comparaison entre filières, les analyses proposées porteront uniquement sur les étudiants de premier cycle. La démonstration s'appuiera sur l'existence de trois segments dans l'enseignement supérieur, aux caractéristiques antagonistes : un segment sélectif, recrutant scolairement et socialement ses étudiants et proposant des conditions d'études favorables à la réussite du plus grand nombre (CPGE, Santé) ; un segment professionnalisant, sélectionnant un petit nombre d'étudiants, qui ne sont pas des héritiers, en vue de les préparer à une insertion professionnelle à court terme (STS, IUT) et assurant une transition aménagée entre le lycée et l'enseignement supérieur (Beaud, 2008) ; un segment universitaire, recrutant massivement des étudiants aux profils variés qui travaillent dans des conditions qui ne permettraient la réussite que de quelques-uns, ceux qui sont parvenus à s'affilier au monde universitaire (Coulon, 1997). Ces derniers seraient plus inquiets pour leur avenir professionnel, a contrario des étudiants inscrits dans les filières sélectives et/ou professionnalisantes.

Indépendamment de sa qualité intrinsèque, l'offre de formation peut donc être distinguée selon son caractère sélectif et/ou professionnalisant, selon la valeur des diplômes pour l'accès aux emplois les plus qualifiés et selon les exigences de la formation. Ces trois perspectives seront analysées successivement. Dans un premier temps, nous insisterons sur le lien existant entre critères de sélection, profils étudiants et attractivité des filières du supérieur. Ensuite, ce sont les représentations de la valeur sociale des diplômes pour l'accès au marché du travail qui seront interrogées. Nous faisons l'hypothèse que dans les filières ouvertes, l'éducation (la suréducation) constitue une stratégie défensive, s'inscrivant en miroir des représentations des faibles débouchés potentiels à l'issue de la formation, a contrario des filières sélectives et professionnalisantes. Enfin, nous montrerons que si les types d'études constituent des cadres de socialisation du monde étudiant, c'est aussi parce qu'ils différencient fortement les pratiques et les emplois du temps studieux, les jugements et les formes de sociabilité étudiante.

Afin de rendre compte de l'hétérogénéité de l'expérience étudiante sous l'angle général du rapport aux études et à l'avenir professionnel, nous mobiliserons la dernière enquête «Conditions de vie » menée par l'Observatoire de la vie étudiante. Cette enquête nationale, conduite en 2006, fournit une information complète et détaillée sur l'articulation entre conditions de vie et déroulement des études (encadré 1).

\section{DES CHOIX D'ORIENTATION DISTINCTIFS OU RESTRICTIFS}

À l'entrée dans l'enseignement supérieur, les étudiants ont déjà un long passé scolaire fait de succès 


\section{Encadré 1}

\section{L'enquête "Conditions de vie des étudiants »}

L'enquête "Conditions de vie » de l'Observatoire national de la vie étudiante (OVE) est effectuée tous les trois ans depuis 1994. Les données présentées ici sont issues principalement de la cinquième et dernière édition, réalisée au printemps 2006.

\section{Questionnaire}

II comprend environ 250 questions, restées pour la plupart identiques de 1994 à 2006. Ces questions permettent de décrire les conditions de vie et d'études, de recueillir les jugements que les étudiants portent à leur égard, de disposer de points de repères sur le cursus et d'établir une signalétique sociodémographique (ressources, diplômes, situation résidentielle, statut conjugal des parents, sexe, âge, taille de la fratrie).

\section{Échantillon}

Le questionnaire est envoyé par voie postale à un échantillon aléatoire d'un quinzième des étudiants d'université (IUT - institut universitaire de technologie - inclus) et remis aux élèves d'un échantillon raisonné de classes supérieures de lycée (CPGE - classes préparatoires aux grandes écoles - et STS - sections de techniciens supérieurs -, publics MESR - ministère de l'Enseignement supérieur et de la Recherche). Le taux de réponse moyen, de l'ordre de $25 \%$, permet de recueillir près de 25000 réponses.

Pour garantir une meilleure représentativité, et corriger les inégalités de taux de réponses selon les catégories étudiantes, les données brutes sont pondérées à partir du nombre d'inscrits dans les établissements, centralisé par la Direction de l'Évaluation, de la Prospective et de la Performance. La grande taille de l'échantillon, la pondération opérée selon des variables majeures (telles que le cycle, le type d'études, le sexe, l'âge, le site...l, la prise en compte dans le contenu et la forme du questionnaire des préoccupations et attitudes de sous-populations étudiantes, habituellement réticentes à l'égard des investigations académiques, permettent d'estimer que l'enquête de l'OVE atteint une bonne représentativité. Les enquêtés représentent ainsi les 1633000 étudiants inscrits au cours de l'année universitaire 20052006 à l'université ou en classes supérieures de lycée publics, soit environ les trois quarts des effectifs de l'enseignement supérieur.

Afin de prendre en compte la diversité des établissements, filières et disciplines, tout en neutralisant l'avancée dans les études, seuls les premiers cycles ont été retenus dans cette analyse ( 18434 étudiants représentant environ 1130000 inscrits). L'importance de l'échantillon enquêté permet d'obtenir un effectif suffisant d'étudiants en premier cycle par type d'études et par grandes filières à l'université : 1278 en CPGE, 1799 en STS, 2280 en IUT, 1636 en Santé, 2821 en Sciences, 3166 en Droit, Économie, 454 en Lettres, SHS (sciences humaines et sociales).

\section{Dimension internationale}

Depuis 1994, l'OVE est intégré au programme Eurostudent qui compare les résultats d'enquêtes menées dans une vingtaine de pays européens sur la situation sociale des étudiants, et permet de dresser un panorama de leurs conditions de vie (Schnitzer, 2008).

ou d'échecs, de sélection et d'orientations. En raison de ces multiples tris scolaires, les filières du supérieur n'accueillent ni ne composent les même profils et génèrent des « laissés pour compte » de la sélection. Malgré les politiques de démocratisation mises en œuvre ces dernières décennies, l'École française reste particulièrement sélective (Baudelot et Establet, 2009), les établissements d'enseignement supérieur se distinguant selon la nature du public et la réputation de l'établissement (Felouzis et Perroton, 2007). L'étude des différences de parcours, de réussite et de caractéristiques des élèves permettrait alors de prévoir l'appartenance à l'une ou l'autre des filières de l'enseignement supérieur.

En France, l'accès à l'enseignement supérieur, après l'obtention du baccalauréat, s'articule à un âge 
d'entrée dans l'enseignement supérieur précoce ${ }^{4}$ et à des parcours de réussite dans l'enseignement secondaire qui déterminent l'appartenance à l'un ou l'autre des segments du supérieur. Dans le segment sélectif, il est plus probable d'avoir obtenu un baccalauréat scientifique avec une mention, sans redoublement préalable (bac à l'heure ou en avance), que dans le segment professionnalisant (tableau 1). Si les possibilités scolaires structurent les parcours et limitent les choix, les seuls critères académiques ne permettent pas de rendre compte des différences d'orientation, marquées en particulier par l'origine sociale ${ }^{5}$. Nombre de travaux ont souligné la polarisation des enfants de cadres dans les filières les plus élitistes, renforcée par la faible ouverture sociale des Grandes écoles (Albouy et Wanecq, 2003 ; Duru-Bellat et Kieffer, 2008). Les enfants de cadres supérieurs, d'industriels et de membres des professions libérales sont surreprésentés dans l'enseignement supérieur, et particulièrement dans le segment sélectif (47\% contre $17 \%$ pour le segment professionnalisant). Le segment sélectif comporte aussi la proportion la plus importante d'étudiants dont les parents ont obtenu un diplôme de l'enseignement supérieur (45\% pour le père dans le segment sélectif contre $28 \%$ dans le segment universitaire).

À l'inverse, les enfants d'employés, d'ouvriers ou d'agriculteurs sont sous-représentés dans l'enseignement supérieur et dans tous les types d'études, à l'exception du segment professionnalisant. La hiérarchie sociale des établissements va des CPGE aux STS. Dans les universités, les différences sociales sont plus atténuées, les profils sociaux plus diversifiés.

Si la représentation des origines sociales reste inégale, la massification a permis une relative ouverture de l'accès à l'enseignement supérieur aux classes populaires. Cependant, l'avantage demeure considérable,

\footnotetext{
${ }^{4}$ La France se caractérise par une quasi-absence de voies non traditionnelles d'accès à l'enseignement supérieur (non traditionnal routes to higher education). En Angleterre, au Pays de Galles, en Ecosse, en Estonie, au Pays-bas et en Slovénie, entre 10 et $15 \%$ des étudiants accèdent à l'enseignement supérieur par le biais d'équivalence (Schnitzer, 2008).

${ }^{5}$ Les « classes populaires » regroupent les enfants d'employés, d'ouvriers ou d'agriculteurs ; les « classes moyennes », les enfants des membres des professions intermédiaires, d'artisans, de commerçants ou de chefs de petites entreprises ; enfin, les « classes supérieures » désignent les enfants de cadres supérieurs, d'industriels, de membres des professions libérales.
}

surtout si l'on précise que les enfants de cadres et professions intellectuelles supérieures ont des parcours d'études dans le supérieur plus longs. On parle à cet égard d'une « démocratisation ségrégative » (Merle, 2000), plus d'études ne signifiant pas les mêmes études pour tous. Dans ce contexte, l'opération de choix des études constitue un facteur clé dans la création et la reproduction de différenciations internes aux institutions d'études supérieures (Ball et alii, 2001 ; Bourdieu, 1979).

En triant un nombre limité d'étudiants spécifiques, la sélection homogénéise les publics du secteur sélectif, assurant ainsi aux filières qui y recourent les conditions d'une adéquation maximale entre les dispositions des étudiants et les exigences de la formation (Millet et Thin, 2007). Au moment d'accéder à l'enseignement supérieur, certains bacheliers seraient alors contraints de renoncer à leur projet initial ou à l'ajuster, car l'entrée dans une formation ou un établissement sélectif leur a été refusée. Le premier cycle universitaire a ainsi pu être considéré comme une zone de relégation attirant des bacheliers en retard et/ou n'étant pas parvenus à intégrer les cursus ayant leur préférence (Berthelot, 1990 ; 1993). En 2006, c'est le cas d'un tiers des étudiants de premier cycle lors de leur primointégration dans l'enseignement supérieur ${ }^{6}$. À mesure que les effectifs étudiants globaux tendent à se stabiliser, voire à diminuer dans l'enseignement supérieur, la proportion d'étudiants contraints de renoncer à leur projet initial diminue également ${ }^{7}(-10$ points de pourcentage entre 1994 et 2006). Les étudiants (les lycéens) parviendraient donc de plus en plus souvent à s'orienter selon leurs choix. Plusieurs analyses sont possibles : soit les formes d'auto-sélection ou d'autolimitation augmentent pour les candidats potentiels aux formations les plus sélectives; soit les formations traditionnellement les plus fermées tendent à s'ouvrir (socialement) ${ }^{8}$; soit les hiérarchies scolaires se

\footnotetext{
${ }^{6}$ Réponse à la question : « Lors de cette première inscription, aviez-vous cherché à entrer sans y parvenir ... en STS; en classe préparatoire ; en IUT ; en IEP ; dans une autre formation sur concours, entretien ou dossier ; dans un autre établissement que celui où vous vous êtes inscrit(e) ; dans une autre discipline que celle dans laquelle vous vous êtes inscrit(e) ?».

7 Lors des différentes vagues de l'enquête triennale de l'OVE, les proportions d'étudiants déclarant avoir tenté d'entrer dans une formation sans y parvenir sont de $45 \%$ en $1994,39 \%$ en 1997 , $41 \%$ en 2000, $39 \%$ en 2003 et $35 \%$ en 2006.

${ }^{8}$ La plupart des formations supérieures réputées les plus sélectives ont mis en place des dispositifs d'ouverture sociale par le biais de
} 
Tableau 1

Caractéristiques des étudiants de 1er cycle selon le type d'études (en \%)

\begin{tabular}{|c|c|c|c|c|c|c|c|c|c|c|}
\hline & \multicolumn{3}{|c|}{ Segment sélectif } & \multicolumn{3}{|c|}{$\begin{array}{c}\text { Segment } \\
\text { professionnalisant }\end{array}$} & \multicolumn{4}{|c|}{ Segment universitaire } \\
\hline & CPGE & Santé & Ensemble & STS & IUT & Ensemble & Sciences & $\begin{array}{c}\text { Droit, } \\
\text { Économie }\end{array}$ & $\begin{array}{l}\text { Lettres, } \\
\text { SHS }\end{array}$ & Ensemble \\
\hline Femme & 43 & 60 & 54 & 49 & 39 & 45 & 38 & 59 & 73 & 60 \\
\hline $\begin{array}{l}\text { Issu des classes } \\
\text { supérieures }\end{array}$ & 50 & 45 & 47 & 14 & 21 & 17 & 29 & 31 & 27 & 29 \\
\hline $\begin{array}{l}\text { Père diplômé de } \\
\text { l'enseignement supérieur }\end{array}$ & 48 & 44 & 45 & 12 & 18 & 14 & 30 & 31 & 26 & 28 \\
\hline $\begin{array}{l}\text { Mère diplômée de } \\
\text { l'enseignement supérieur }\end{array}$ & 38 & 29 & 32 & 9 & 14 & 11 & 20 & 22 & 22 & 22 \\
\hline $\begin{array}{l}\text { Titulaire d'un bac. } \\
\text { scientifique }\end{array}$ & 70 & 88 & 82 & 11 & 37 & 22 & 73 & 17 & 13 & 30 \\
\hline Mention au bac. & 87 & 60 & 70 & 39 & 45 & 41 & 44 & 42 & 42 & 42 \\
\hline $\begin{array}{l}\text { Bac. à l'heure ou en } \\
\text { avance }\end{array}$ & 93 & 82 & 86 & 41 & 58 & 48 & 66 & 58 & 58 & 60 \\
\hline
\end{tabular}

Source : OVE, Enquête Conditions de vie 2006.

Champ : Étudiants inscrits l'année universitaire 2005-2006 en premier cycle universitaire (France métropolitaine + DOM-COM) et en classe supérieure de lycée (CPGE et STS, publics MEN-MESR, France métropolitaine) ( $\mathrm{n}=18$ 434).

Sigles : CPGE : classes préparatoires aux grandes écoles ; STS : sections de techniciens supérieurs ; IUT : instituts universitaires de technologie ; SHS : sciences humaines et sociales.

Note de lecture : $50 \%$ des élèves inscrits en CPGE en 2005-2006 sont issus des classes supérieures contre $14 \%$ des élèves de STS.

recomposent ${ }^{9}$. Une analyse des types de renoncement et des profils des « renonceurs », associée à celle des évolutions de la structure de l'enseignement supérieur (présentées en introduction), laisse à penser que ces trois pistes explicatives s'articulent.

Si l'on considère qu'un étudiant effectue un choix par défaut lorsqu'il s'inscrit dans une filière à la suite d'une tentative avortée d'entrer dans une (autre) formation sélective, il est intéressant de mesurer dans quelle proportion chaque type de formation accueille ces « laissés pour compte» de la sélection. L'hypothèse consistant à considérer que les formations les plus prestigieuses sont privilégiées par une majorité d'étudiants et que la proportion de « relégués » est la plus importante dans les formations non sélectives (donc universitaires), s'avère, au regard des données recueillies, invalidée. Les élèves des formations sélectives sont en proportion plus nombreux

conventions. Sciences Po a joué un rôle pionnier en ce domaine en créant, dès 2000-2001, une procédure d'admission réservée aux lycéens scolarisés en zone d'éducation prioritaire.

${ }^{9}$ Nous verrons ultérieurement que le passage en IUT (et plus marginalement en STS) est envisagé le plus souvent comme une étape dans un parcours studieux au long cours. (par rapport aux étudiants d'université) à avoir été contraints de renoncer à leur projet initial : $49 \%$ des élèves de STS, $43 \%$ des élèves d'IUT et $34 \%$ des élèves de CPGE contre $31 \%$ des étudiants de premier cycle universitaire. Les renoncements s'opèrent principalement au sein des mêmes types de filières mais dans des établissements et/ou des disciplines différents. En particulier, les étudiants qui ont intégré une filière professionnalisante avaient fréquemment tenté d'accéder au même type de formation, sans doute dans un établissement plus prestigieux que celui auquel ils ont finalement eu accès. Les étudiants d'université ont, quant à eux, plus rarement tenté d'accéder aux filières sélectives de l'enseignement supérieur (moins d'un étudiant sur trois). Faut-il alors considérer une moindre relégation à l'université ? Ou au contraire une plus forte auto-sélection des étudiants d'université ? À l'instar de Ball et alii (2005), on peut considérer que le choix des études se situe au sein de deux registres de sens et d'action : l'un « cognitif et performatif», ayant trait à «l'adéquation entre les résultats et la sélection dans les institutions de formations »; l'autre « social/culturel», lié «aux classifications de soi et des institutions ». En d'autres termes, dans un 
contexte de hiérarchie implicite, les choix d'orientation peuvent être soit stratégiques et distinctifs pour les plus informés (Duru-Bellat et Merle, 1997), soit restrictifs pour les autres, en particulier les enfants des milieux populaires. Ceci expliquerait la non-significativité de la variable d'origine sociale sur la probabilité d'avoir échoué à l'entrée dans une (autre) formation ou un (autre) établissement sélectif ${ }^{10}$, a contrario des variables de réussite scolaire ${ }^{11}$.

\section{LE PROJET, POUR S'AJUSTER AUX REPRÉSENTATIONS DU MARCHÉ DU TRAVAIL}

L'orientation dans un parcours d'études s'articule aussi à l'existence d'un projet (scolaire et/ou professionnel) entendu comme "représentation subjective de l'utilité des études pour un acteur capable de définir des objectifs, d'évaluer des stratégies et leur coût » (Dubet, 1994). Cette utilité est évaluée en particulier à partir du diplôme visé (niveau et type d'études) et de sa supposée rentabilité sur le marché du travail, dans un contexte de dégradation des perspectives de mobilité sociale des jeunes générations (Peugny, 2009). Si les individus investissent dans l'éducation, c'est entre autre parce qu'elle peut être rentabilisée sur le marché du travail, le diplôme constituant le principal signal, pour les employeurs, du potentiel des individus en début de vie active. Plus le niveau d'études est élevé, meilleure est l'insertion, quel que soit l'indicateur retenu : taux de chômage, contrat de travail, salaire, accès à l'emploi qualifié (Calmand et Hallier, 2008 ; Giret et alii, 2003). Le

\footnotetext{
${ }^{10}$ C'est ce que confirme également l'analyse à partir d'un modèle de régression logistique, opérée sur la probabilité d'avoir tenté d'accéder à au moins une formation ou un établissement sans y parvenir. À parcours scolaire équivalent (bac, mention, âge au bac, type d'études) et à caractéristiques individuelles identiques, la probabilité de renoncer à au moins un type d'études ou d'établissement ne dépend pas de l'origine sociale (non significative au seuil de $10 \%$ ).

11 Le fait d'avoir obtenu une mention très élevée au baccalauréat limite les renoncements $(38 \%$ des élèves n'ayant pas obtenu de mention au bac ont tenté sans succès d'entrer dans une autre formation, contre $25 \%$ des élèves ayant obtenu la mention « Très bien »), alors que l'obtention d'un bac professionnel les accrô̂t (51\%, contre $27 \%$ des bacheliers littéraires et $29 \%$ des bacheliers scientifiques).
}

(niveau de) diplôme a une valeur instrumentale forte sur le marché du travail et l'aspiration à un niveau d'études toujours plus élevé constitue un comportement rationnel de la part des étudiants, dont la généralisation n'est pas sans effet pervers (Duru-Bellat, 2006 ; Passeron, 1982). Rien de surprenant alors que les trois quarts des étudiants de $1^{\text {er }}$ cycle envisagent de poursuivre leurs études au moins jusqu'au niveau master ${ }^{12}$. L'aspiration à des études plus longues est également le fruit de transformations institutionnelles. En particulier, l'évolution de l'architecture des études autour de trois grades principaux ${ }^{13}$ a conduit au report de l'obtention des premiers diplômes universitaires (du DEUG - diplôme d'études universitaires générales - vers la licence, de la maîtrise vers le master). À l'université, les niveaux bac +2 et bac +4 ne sont visés que par une minorité d'étudiants de premier cycle (respectivement $2 \%$ et $7 \%$ en 2006 contre $18 \%$ et $19 \%$ en 1997).

La comparaison des projets d'études selon l'appartenance à un type d'études présente un intérêt descriptif évident. Toutefois, elle ne permet pas d'imputer, sans précaution, les différences constatées, par exemple à l'origine sociale des étudiants des différents segments de l'enseignement supérieur. Si la comparaison est pertinente lorsque les populations sont homogènes, il n'en est rien lorsque les sous-populations diffèrent en termes de parcours scolaire, d'âge, de sexe, de milieu social (tableau 1). Pour apprécier le poids spécifique de chacune de ces caractéristiques, tout en neutralisant les effets respectifs des autres variables en jeu, nous avons mesuré, grâce à des modèles de régression logistique, les probabilités, pour ces étudiants de premier cycle, de viser un niveau d'études supérieur ou égal au master et d'être inquiets vis-à-vis de l'avenir professionnel (tableau 2).

On constate ainsi que, toutes choses égales par ailleurs, il est beaucoup plus probable pour un élève du segment sélectif (OR - odd ratio - de 5,9 pour les étudiants de CPGE) de s'engager dans un parcours

\footnotetext{
12 Dans les faits, l'aspiration généralisée à des études plus longues ne se concrétise pas systématiquement par une réussite dans le supérieur : $19 \%$ des « sortants » de l'enseignement supérieur de la «Génération 2004 » obtiennent au moins un diplôme de niveau master, alors que $20 \%$ quittent l'enseignement supérieur sans diplôme (Calmand et Hallier, op. cit.).

${ }^{13}$ La réforme remplace le schéma d'études $2 / 1+1 / 1+3$, issu du plan Fouchet de 1966, par un schéma 3/2/3.
} 
Tableau 2

Probabilité estimée... (1)

\begin{tabular}{|c|c|c|}
\hline & $\begin{array}{l}\text { de viser un niveau d'études } \\
\text { supérieur ou égal à bac }+5\end{array}$ & 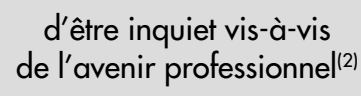 \\
\hline \% d'étudiants concernés & $66,7 \%$ & $28,1 \%$ \\
\hline \multicolumn{3}{|l|}{ Sexe } \\
\hline Masculin & 1,5 & n.s. \\
\hline Féminin & 1,0 & 1,0 \\
\hline \multicolumn{3}{|l|}{ Origine sociale } \\
\hline Classes populaires & 1,0 & 1,0 \\
\hline Classes moyennes & 1,4 & 0,9 \\
\hline Classes supérieures & 2,1 & 0,8 \\
\hline \multicolumn{3}{|l|}{ Âge au Bac } \\
\hline Bac en avance & 1,3 & n.s. \\
\hline Bac à l'heure & 1,0 & 1,0 \\
\hline Bac en retard & 0,6 & n.s. \\
\hline \multicolumn{3}{|l|}{ Mention au Bac } \\
\hline Passable, pas de mention & 1,0 & 1,0 \\
\hline Assez bien & 1,4 & n.s. \\
\hline Bien & 1,9 & 0,8 \\
\hline Très bien & 2,4 & 0,7 \\
\hline \multicolumn{3}{|c|}{ Activité rémunérée pendant l'année universitaire } \\
\hline Oui & n.s. & n.s. \\
\hline Non & 1,0 & 1,0 \\
\hline \multicolumn{3}{|l|}{ Type d'études suivies } \\
\hline Lettres, SHS & 1,0 & 1,0 \\
\hline Droit, Économie & n.s. & 0,4 \\
\hline Sciences & n.s. & 0,6 \\
\hline Santé & n.s. & 0,0 \\
\hline IUT & 0,3 & 0,2 \\
\hline STS & 0,1 & 0,3 \\
\hline CPGE & 5,9 & 0,2 \\
\hline \multicolumn{3}{|c|}{ Heures de présence aux cours durant une semaine } \\
\hline Aucune & 0,7 & 1,8 \\
\hline Moins de $15 \mathrm{~h}$ & 0,7 & 1,8 \\
\hline De 15 à moins de $22 \mathrm{~h}$ & n.s. & 1,4 \\
\hline De 22 à moins de $30 \mathrm{~h}$ & 1,1 & 1,3 \\
\hline $30 \mathrm{~h}$ et plus & 1,0 & 1,0 \\
\hline
\end{tabular}


(Suite tableau 2)

\begin{tabular}{|c|c|c|}
\hline \multicolumn{3}{|l|}{ Niveau d'études visé } \\
\hline $\mathrm{Bac}+1 \mathrm{a} \mathrm{Bac}+2$ & & 1,6 \\
\hline $\mathrm{Bac}+3$ & & 1,6 \\
\hline $\mathrm{Bac}+4$ & & 1,3 \\
\hline$B a c+5$ & & 1,0 \\
\hline Bac +6 et plus & & n.s. \\
\hline \multicolumn{3}{|c|}{ Investissement dans les études ${ }^{[3]}$} \\
\hline Oui & 1,4 & n.s. \\
\hline Non & 1,0 & 1,0 \\
\hline \multicolumn{3}{|c|}{ Jugement de la facilité à trouver du travail avec sa formation } \\
\hline Difficilement & 0,5 & \\
\hline Plutôt difficilement & 0,7 & \\
\hline Plutôt facilement & 1,0 & \\
\hline Très facilement & n.s. & \\
\hline Rapport de vraisemblance ${ }^{(4)}$ & $4228,03(<.0001)$ & $2362,73(<.0001)$ \\
\hline
\end{tabular}

Les modalités de référence sont en italique; (n.s. : non significatif au seuil de $5 \%$ ).

(1) Les résultats des odds ratio sont présentés ci-dessus. L'odds ratio, ou rapport de chance, vaut 1 en cas d'indépendance statistique entre les deux variables dichotomiques. On a affecté la valeur 1 à la variable explicative choisie par convention comme situation de référence. Quand l'appartenance à l'une des variables qui font partie de la dimension explicative considérée augmente la probabilité de présenter le comportement étudié, la valeur de l'odds ratio est supérieure à 1 ; lorsqu'elle la diminue, toujours par rapport à la situation de référence, elle est inférieure à 1 . On a choisi comme situation de référence la situation modale. Les régressions ont été calculées sur l'échantillon pondéré (pondérations normalisées).

(2) Sont considérés comme inquiets, les étudiants qui pensent qu'à l'issue de leur formation ils trouveront difficilement ou plutôt difficilement du travail.

(3) Sont considérés comme investis, les étudiants qui effectuent 15 heures ou plus de travail personnel par semaine et dont la moitié des lectures concerne les études.

(4) La valeur du rapport de vraisemblance (likelihood ratio) correspond à la différence entre la -2Log de la vraisemblance du modèle vide et la -2 Log de vraisemblance du modèle avec les dimensions explicatives. Cette valeur est ensuite comparée au Chi ${ }^{2}$, pour le nombre de degrés de liberté ou de modalités explicatives introduites dans le modèle. Le résultat $<.0001$ indique que le modèle avec les dimensions explicatives est significativement meilleur que le modèle vide. Cela signifie qu'au moins une des dimensions explicatives a un impact significatif sur le modèle.

Source : OVE, Enquête « Conditions de vie $2006 »$.

Champ : Étudiants inscrits l'année universitaire 2005-2006 en premier cycle universitaire (France métropolitaine + DOM-COM) et en classe supérieure de lycée (CPGE et STS, publics MEN-MESR, France métropolitaine) $(\mathrm{n}=15635)$

Note de lecture : Le groupe de personnes de référence est celui par rapport auquel l'ensemble des résultats sont interprétés (étudiante, en Lettres et SHS, qui a obtenu son baccalauréat à l'âge de 18 ans, qui n'exerce pas d'activité rémunérée pendant ses études, etc.). Les élèves de CPGE, qui par ailleurs présentent l'ensemble des caractéristiques de la personne de référence, ont la probabilité la plus élevée de viser un niveau d'études supérieur ou égal à bac +5 (Odds ratio de 5,9). On rend compte ainsi de l'effet spécifique du type d'études, toutes choses égales par ailleurs.

Sigles : CPGE : classes préparatoires aux grandes écoles ; STS : sections de techniciens supérieurs ; IUT : instituts universitaires de technologie ; SHS : sciences humaines et sociales.

d'études de niveau supérieur ou égal au master que pour un étudiant du segment universitaire (OR de 1 pour les étudiants de Lettres, $\mathrm{SHS}^{14}$ ) ou professionnalisant (OR de 0,1 pour les étudiants de STS et de 0,3 pour ceux d'IUT). Pour ces derniers, soulignons

${ }^{14}$ Lettres, Sciences humaines et sociales. toutefois que l'offre de formation qui s'est développée au sein même des filières universitaires, les licences ${ }^{15}$ et masters professionnels, les a conduits à envi-

${ }^{15}$ Les licences professionnelles, créées en 1999, ont vu leur effectif augmenter en moyenne de $55 \%$ par an et le nombre d'étudiants de $58 \%$ par an (Giret, op.cit) et plus de la moitié des jeunes de la Génération 2004 ayant une licence professionnelle viennent de BTS (Calmand et Hallier, op. cit.). 
sager des cursus plus longs. En particulier, pour les étudiants d'IUT, le niveau bac +2 constitue rarement le terme visé : il n'est envisagé que par $8 \%$ d'entre eux contre $45 \%$ pour le niveau master. La multiplication de ces filières dans les universités participe donc d'une " véritable inflation de l'offre de formation » (Rey, 2009).

Le type d'études, qui sous-tend assez logiquement des parcours différenciés dans l'enseignement supérieur, ne doit pas masquer l'influence des variables sociodémographiques et scolaires. Ainsi, les filles, qui réussissent pourtant mieux dans leur parcours scolaire et de premier cycle universitaire, cèdent, aux paliers les plus sélectifs, la prééminence aux garçons (Gruel, 2006), leur part diminuant en $3^{\text {e }}$ cycle. La probabilité de se projeter dans des études longues (bac +5 et plus) est plus élevée pour les garçons (OR de 1,5) que pour les filles. Les écarts de représentation des groupes sociaux aux niveaux les plus élevés des hiérarchies scolaires s'articulent également à des ambitions variables, les étudiants des catégories supérieures ayant une probabilité plus élevée de se projeter dans des études longues (OR de 2,1). Enfin, le fait d'être engagé dans un parcours de réussite (mention au baccalauréat, baccalauréat en avance) est un facteur favorisant ce type de projet d'études.

Ces stratégies éducatives ne sont pas sans incidence sur les représentations de l'avenir, la probabilité d'être inquiet vis-à-vis de l'avenir professionnel étant d'autant plus faible que le niveau d'études visé est élevé (tableau 2). Les étudiants de premier cycle considèrent dans leur grande majorité $(71 \%)$ que leur formation leur permettra de trouver (très) facilement du travail. Cet optimisme déclaré a même augmenté, puisqu'en 1994 et 1997, ils étaient $61 \%$ à le partager. Les étudiants inscrits dans le segment sélectif sont parmi ceux qui se déclarent les plus optimistes par rapport à leurs perspectives professionnelles (plus de $90 \%$ ). Dans le segment professionnalisant, la part de ceux qui pensent trouver « très facilement » ou « plutôt facilement » un emploi a également augmenté de 1994 à 2006, passant, pour les élèves de STS, de $56 \%$ à $76 \%$ et pour les étudiants d'IUT, de $69 \%$ à $85 \%$. L'évolution est plus contrastée pour les étudiants de premier cycle des différentes filières de l'université. En Sciences et surtout en Droit et Économie, les perspectives se sont nettement améliorées. En Lettres, SHS, elles ont légèrement diminué ${ }^{16}$. Indépendamment des conditions d'études et de réussite dans le secondaire, les étudiants de $1^{\text {er }}$ cycle universitaire connaissent une probabilité beaucoup plus élevée d'être inquiets pour leur avenir que ceux du segment professionnalisant (OR de 1 contre 0,3 en STS et 0,2 en IUT).

Plus l'inquiétude par rapport à l'avenir est importante, plus le secteur public apparaît comme une perspective professionnelle ${ }^{17}$, en particulier pour les étudiants de Lettres, SHS ( $43 \%$ d'entre eux souhaitent y trouver un emploi). Si cette préférence concerne plus massivement les étudiants du segment universitaire, c'est aussi parce que le secteur public constitue un débouché plus important pour ces derniers. Alors que la part des emplois dans ce secteur s'établit autour de $10 \%$ en 2004 pour les titulaires d'un BTS (brevet de technicien supérieur) ou d'un DUT (diplôme universitaire de technologie) (sortis de formation en 2001), elle est nettement plus importante pour les diplômés d'université (38\% après un DEUG, $46 \%$ après une licence) (Giret et alii, 2006). La décrue progressive des débouchés dans le secteur public n'a cependant influé que faiblement sur la part des étudiants de premier cycle désireux d'y trouver un emploi (35\% en 1994 et $31 \%$ en 2006), hormis pour les élèves de CPGE.

La valeur attachée au diplôme par les étudiants diffère sensiblement selon les cursus ; un diplôme n'en vaut pas un autre et n'offre pas les mêmes perspectives. Au-delà du diplôme, les études engagées (en supposant qu'elles soient poursuivies jusqu'à leur terme) conduisent plus ou moins efficacement les étudiants vers le monde du travail et les degrés de certitude ou d'incertitude associés à l'avenir d'une filière d'études constituent une dimension essentielle du rapport aux études. Soulignons que ces inégalités perçues de rendement des filières ne peuvent être comprises indépendamment des cadres organisationnels et d'apprentissage qu'elles proposent.

\footnotetext{
${ }^{16}$ En 2004, le taux de chômage des diplômés de master 1 ou de master 2 de Lettres, SHS était de $15 \%$ alors qu'il était en moyenne de 5 points moins élevé dans les disciplines de Droit et d'Économie (Giret, op. cit.)

$1735 \%$ des étudiants qui pensent trouver difficilement du travail à l'issue de leur formation souhaiteraient travailler dans le secteur public, contre $28 \%$ de ceux qui pensent trouver très facilement du travail.
} 


\section{DES SOCIALISATIONS DISTINCTES SELON LES TYPES D'ÉTUDES}

La composition différenciée des publics de l'enseignement supérieur ainsi que la variabilité des aspirations s'articulent à des variations des pratiques du travail studieux. Les types d'études et la nature spécifique des savoirs transmis forment des cadres d'apprentissage et de socialisation dans des contextes spécifiques : effectifs restreints, encadrement strict et apprentissage dans les segments sélectif et professionnalisant, public plus large et hétérogène bénéficiant de moins de contraintes dans le segment universitaire. Les pratiques des étudiants au sein d'une même filière ne sont pas homogènes mais les filières d'études peuvent engendrer « des types de pratiques intellectuelles, des manières de travailler, des représentations du travail [...] assez différents et relativement indépendants des conditions sociales d'origine des étudiants » (Millet, 2003).

Selon le type d'études, le cadre de vie et le rythme imposé par les études ne sont pas les mêmes. Le rapport entre temps disponible et volume de travail à réaliser pour les études est plus ou moins équilibré. L'étude des comportements studieux fait apparaître une opposition entre les formations universitaires (Amrous, 2005) et celles à plus fort encadrement pédagogique et à effectif restreint (graphique 1). Dans les premières, les étudiants ont globalement moins d'heures de cours et sont moins assidus. Ainsi, l'absentéisme par choix ${ }^{18}$ est particulièrement faible dans les formations les plus encadrées (93\% des élèves de CPGE déclarent ne manquer les cours qu'exceptionnellement ou jamais) par opposition aux formations universitaires (69\% des étudiants à l'université). En dehors des enseignements, le temps de travail personnel oppose les étudiants du segment sélectif à ceux du segment professionnalisant, les premiers y consacrant près de deux fois plus de temps. Les niveaux d'exigence, de structuration de l'emploi du temps, d'investissement distinguent les étudiants appartenant à chaque type d'études et confirment que «tous les étudiants ne sont pas étudiants au même degré » (Grignon et Gruel, 1999).

${ }^{18}$ Il s'agit de la réponse donnée à la question : «Vous arrive-t-il de ne pas assister à des enseignements (cours, TD (travaux dirigés), TP (travaux pratiques ou ateliers)) par choix ? »
Il est toutefois difficile de parler d'investissement studieux en se basant uniquement sur le temps de travail personnel. En effet, en observant les effets de l'appartenance à un type d'études, on enregistre l'effet conjugué d'un ensemble d'autres variables, en particulier le degré d'encadrement pédagogique et le travail scolaire exigé.

Si on distingue les étudiants selon qu'ils effectuent 15 heures (ce qui correspond à la moyenne) ou plus de travail personnel par semaine et qu'au moins la moitié de leurs lectures sont liées aux études, on obtient un autre indice d'investissement studieux ${ }^{19}$ qui concerne un tiers de la population étudiante. En raisonnant toutes choses égales par ailleurs, il est plus probable qu'un élève du segment sélectif adopte ce type de comportement qu'un étudiant du segment universitaire ou professionnalisant, en particulier de Sciences, d'IUT et de STS. Quel que soit le type d'études, plus l'étudiant a un nombre d'heures de cours élevé et plus il a de chances de travailler pour ses études en dehors de ses enseignements. À l'inverse, plus l'étudiant a d'activités en dehors des cours, de loisirs ou exerce un travail rémunéré, et moins il consacre de temps à ses études. Ces types de comportements studieux, en particulier les modes de répartition horaire, peuvent être considérés comme des préfigurations des modes de vie et de travail ultérieurs (Lahire, 1997), avec en pôles opposés ceux qui anticipent une vie sociale fondée sur la nette distinction entre « travail professionnel » et « loisirs » (les élèves du segment professionnalisant) et ceux qui vivent l'apprentissage de rythmes temporels qui préfigurent des lignes de partage plus floues entre ce qui relève du « travail » et ce qui est de l'ordre de la « vie privée » et du « loisir » (les étudiants du segment sélectif).

Selon le type d'études, l'emprise de la vie studieuse est donc plus ou moins forte et exerce une socialisation plus ou moins explicite. C'est aussi ce que révèle l'évaluation par l'étudiant de son cadre d'études, tant au niveau relationnel que matériel. Derrière la satis-

\footnotetext{
${ }^{19}$ Cet indice ne se défait pas totalement de l'influence des contraintes inhérentes au type d'études puisqu'il distingue, au sein de la population étudiante, ceux pour lesquels un travail personnel important est attendu et ceux pour qui l'acquisition de savoirs passe davantage par la lecture. Cependant, il distingue aussi les étudiants qui fournissent un effort personnel de travail en dehors des cours et permet ainsi de comprendre certains mécanismes de l'investissement studieux en dehors des études.
} 
Types d'études et durée du travail studieux (en heures)

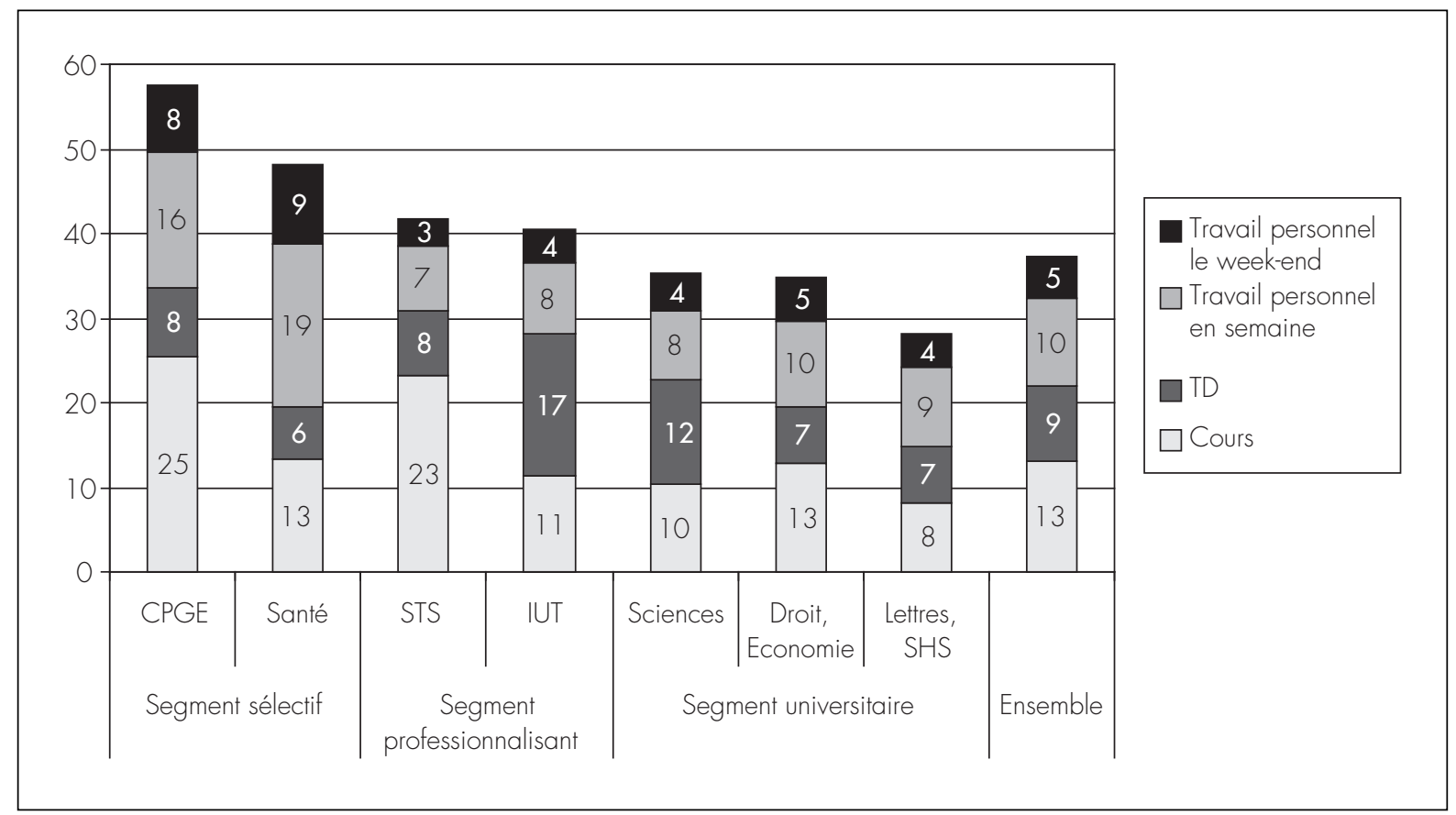

Source : OVE, Enquête « Conditions de vie 2006 ».

Champ : Étudiants inscrits l'année universitaire 2005-2006 en premier cycle universitaire (France métropolitaine + DOM-COM) et en classe supérieure de lycée (CPGE et STS, publics MEN-MESR, France métropolitaine) $(\mathrm{n}=18$ 434)

Note de lecture : Ce sont les étudiants en Santé qui ont le temps de travail personnel le plus élevé avec 28 heures (19 heures en semaine et 9 heures le week-end) et ceux de STS le plus faible avec 10 heures.

Sigles : CPGE : classes préparatoires aux grandes écoles ; STS : sections de techniciens supérieurs ; IUT : instituts universitaires de technologie ; SHS : sciences humaines et sociales.

faction et l'insatisfaction, on perçoit des conditions d'études très différentes, en termes de prestige, d'encadrement pédagogique, de taille et d'équipement des établissements, etc. D'un type d'études à l'autre, on identifie un cadre cognitif spécifique avec des propriétés pédagogiques, des savoirs et des exigences qui ne supposent pas les mêmes techniques ni les mêmes représentations du travail scolaire ou universitaire. Ainsi, les évaluations portées par les étudiants sur leurs conditions d'études opposent principalement les pratiques du segment universitaire à celles des autres secteurs.

Dans l'ensemble, les étudiants les moins satisfaits sur tous les aspects de la vie de l'établissement (matériels, pédagogiques, relationnels ou informationnels) sont les étudiants du segment universitaire. Concernant la dimension pédagogique, ces derniers se montrent plus critiques : enseignants-chercheurs pas assez dispo- nibles (33\% de satisfaits contre $47 \%$ dans le segment sélectif et professionnalisant, $74 \%$ pour les élèves de CPGE), utilité et cohérence des enseignements peu évidentes ( $26 \%$ contre $45 \%$ dans le segment sélectif, $63 \%$ en CPGE), équipements pédagogiques (laboratoires de langues, informatique) insuffisants (31\% contre $41 \%$ dans le segment professionnalisant, $51 \%$ en IUT) et conditions d'organisation et de surveillance des examens peu appréciées ( $29 \%$ contre $53 \%$ dans le segment sélectif ).

$\mathrm{Au}$ contraire, les étudiants du segment professionnalisant et ceux de $\mathrm{CPGE}^{20}$, dans des classes à faibles effectifs, avec des emplois du temps fixes et conséquents déclarent être en contact plus étroits et fréquents avec leurs enseignants. De façon générale,

${ }^{20}$ De ce point de vue, les formations de Santé et les CPGE se distinguent. 
ces étudiants des formations les plus encadrées valorisent la dimension pédagogique de leur formation ${ }^{21}$. Ils la valorisent d'autant plus qu'elle a pu être un élément déterminant dans le choix de leur formation (Beaud, op. cit.) avec comme repoussoir l'organisation des études universitaires. À l'université, le poids du nombre et l'hétérogénéité des étudiants ont infléchi la situation pédagogique et l'environnement humain de l'université, l'image dominante étant celle d'un monde atomisé et «désenchanté » (Dubet, op. cit. ; Musselin, 2001).

Plus ou moins volumineux, contraignants et structurés, les emplois du temps institutionnels produisent des effets de socialisation sur le travail universitaire mais aussi sur la façon dont l'étudiant mène sa vie en dehors des cours, perçoit ses études et son avenir. Plus le rythme imposé par les études est important, toutes choses égales par ailleurs, plus les individus sont en confiance quant à leur possibilité d'insertion à l'issue de leur formation (tableau 2). Un emploi du temps studieux très contraignant limite les latitudes des étudiants et réduit les possibilités d'exercer, par exemple, une activité rémunérée ou certains comportements non studieux. Les étudiants du segment universitaire occupent plus fréquemment que les autres des emplois qui entrent en concurrence avec les études ${ }^{22}: 14 \%$ contre $4 \%$ dans le segment professionnalisant et $2 \%$ dans le segment sélectif. Selon les filières d'études, les étudiants disposent d'un temps libre de toute astreinte scolaire plus ou moins grand. Ceux qui ont des emplois du temps très chargés doivent faire face à la rareté du temps disponible pour le travail personnel. À l'inverse, ceux qui bénéficient d'une abondance de temps libre doivent savoir le gérer et ne pas « décrocher » (Beaupère et alii, 2007 ; Beaupère, Boudesseul et Macaire, 2009). L'influence des éléments extérieurs est alors plus importante et l'arbitrage entre études et vie en dehors des études est posé.

\footnotetext{
${ }^{21}$ Parmi les formations les plus « encadrées », les élèves de STS sont les moins satisfaits $(43,3 \%$ de satisfaits ou très satisfaits) sur ce point. Ils se révèlent également plus insatisfaits de l'information concernant l'orientation et les débouchés (53\% d'insatisfaits contre $32 \%$ pour les élèves de CPGE).

${ }^{22}$ Activités exercées au moins six mois par an et au moins à mi-temps pendant l'année universitaire, à l'exception de celles qui sont intégrées aux études.
}

Avec près d'un million cent mille inscrits en premier cycle à l'université ou en classes supérieures de lycée, il paraît évident qu'il n'existe pas un profil-type d'étudiants dont les caractéristiques résumeraient celles de l'ensemble. Cependant, il n'y a pas non plus « une infinité d'individus dont la singularité serait irréductible » (Gruel, 2009). Les différences entre étudiants s'organisent notamment sur la base de l'appartenance à un secteur d'études (sélectif, professionnalisant ou universitaire). Au sein des parcours, qu'ils soient reconstitués (orientation post-baccalauréat), présents (pratiques studieuses, conditions d'études actuelles) ou anticipés (projets d'études et professionnels), des oppositions existent en matière de comportement et de caractéristiques des étudiants.

Les étudiants ici analysés (inscrits en $1^{\text {er }}$ cycle) commencent leur carrière estudiantine et au-delà professionnelle. L'analyse de leurs anticipations permet de souligner combien les types d'études constituent des cadres de socialisation déterminants, neutralisant partiellement le poids des dispositions intervenues lors du tri scolaire en amont des études supérieures. L'hétérogénéité des conditions d'études conduit alors à considérer l'enseignement supérieur comme un espace segmenté sur lequel l'obtention de références universitaires rares constitue un enjeu entre groupes sociaux (Collins, 1979), permettant à une partie de la population de se protéger contre l'insécurité et/ou l'incertitude professionnelle.

Une telle approche permet de souligner les limites de la massification et de la démocratisation de l'enseignement supérieur. En particulier, elle montre que la diversification des structures d'enseignement supérieur et des pratiques sélectives tend à restreindre les possibilités réelles d'égalisation des chances. Toutefois, une analyse en termes de segmentation accorde insuffisamment d'intérêt aux ressources et compétences que les individus peuvent développer dans des « institutions faibles » (Felouzis, 2001). On sait notamment qu'il existe des combinaisons réussies entre la sphère des études et d'autres formes d'engagement : professionnels, associatifs, de mobilité, conjugaux... 


\section{Bibliographie}

Albouy V., Wanecq T. (2003), " Les inégalités sociales d'accès aux grandes écoles ", Économie et Statistique, n 361 , pp. 27-52.

Amrous N. (2005), « La disparité des emplois du temps », OVE Infos, $\mathrm{n}^{\circ} 11$.

Ball S. J., Davies J., David M., Reay D. (2001), "Décisions, différenciations et distinctions : vers une sociologie du choix des études supérieures ", Revue française de pédagogie, $\mathrm{n}^{\circ} 136$, juillet-aoûtseptembre, pp. 65-75.

Baudelot C., Establet R. (2009), L'élitisme républicain, Seuil, Collection « République des Idées ».

Beaud S. (2008) «Enseignement supérieur : la "démocratisation scolaire" en panne ", Formation Emploi, $\mathrm{n}^{\circ} 101$, pp. 149-165.

Beaupère N., Chalumeau L., Gury N., Hugrée C. (2007), L'abandon des études supérieures, Paris, La Documentation française, Collection « Panorama des savoirs $»$.

Beaupère N., Boudesseul G., Macaire S. (2009), « Sortir sans diplôme de l'université. De l'orientation post-bac à l'entrée sur le marché du travail », OVE Infos, $\mathrm{n}^{\circ} 21$.

Berthelot J.-M. (1990), « Les effets pervers de l'expansion des enseignements supérieurs : le cas de la France », Sociétés contemporaines, $\mathrm{n}^{\circ}$ 4, pp. 109-122.

Berthelot J.-M. (1993), École, orientation, société, Paris, PUF.

Bourdieu P. (1979), La distinction ; Critique sociale du jugement, Éditions de Minuit.

Calmand J., Hallier P. (2008), « Être diplômé de l'enseignement supérieur, un atout pour entrer dans la vie active », Céreq, $B r e f \mathrm{n}^{\circ} 253$.

Collins H. (1979), The Credential society, Academic Press.

Coulon A. (1997), Le métier d'étudiant, PUF.
Desmaziere-Berlie C. (1999), «La sélection à l'entrée de l'IUT : réputation, réalité et effet », L'orientation scolaire et professionnelle $28, n^{\circ} 4$.

Dubet F. (1994), « Dimensions et figures de l'expérience étudiante dans l'université de masse ", Revue française de sociologie, $\mathrm{n}^{\circ}$ 35/4, pp. 511-532.

Duru-Bellat M. (2006), L'inflation scolaire. Les désillusions de la méritocratie, Éditions du Seuil.

Duru-Bellat M., Kieffer A. (2008), « Du baccalauréat à l'enseignement supérieur : déplacement et recomposition des inégalités ", Population, vol. $63, \mathrm{n}^{\circ} 1$, pp. 123-157.

Duru-Bellat M., Merle P. (1997), « La démocratisation impossible ? Usages sociaux de l'école et inégalités sociales des cursus scolaires ", Savoir, Éducation-Formation, 3-4, pp. 3-25.

Duru-Bellat M. et Verley E. (2009), « Les étudiants au fil du temps : contexte et repères institutionnels » in Gruel L., Galland O., Houzel G. (sous la direction de), Les étudiants en France. Histoire et sociologie d'une nouvelle jeunesse, Rennes, PUR, novembre, pp. 195-219.

Felouzis G. (2001), La condition étudiante. Sociologie des étudiants et de l'université, Paris, PUF.

Felouzis G., Perroton J. (2007), « Les marchés scolaires » : une analyse en termes d'économie de la qualité », Revue française de sociologie, vol. 48, 4, pp. 693-722.

Galland O., Oberti M. (1996), Les étudiants, Paris, La Découverte.

Giret J.-F., Moullet S., Thomas G. (2003), « De l'enseignement supérieur à l'emploi : les trois premières années de vie active de la génération 98 », Notes Emploi Formation, $\mathrm{n}^{\circ}$ 1, Céreq, juin.

Giret J.-F., Molinari-Perrier M., Moullet S. (2006), « 2001-2004 : les sortants de l'enseignement supérieur face au marché du travail ", Notes Emploi Formation, ${ }^{\circ} 21$, Céreq, mars. 
Giret J.-F. (2009), « L'évolution des conditions d'insertion professionnelle des étudiants ", in Gruel L., Galland O., Houzel G. (sous la direction de), Les étudiants en France. Histoire et sociologie d'une nouvelle jeunesse, Rennes, PUR, novembre, pp. 331-348.

Grignon C., Gruel L. (1999), La vie étudiante, Paris, PUF.

Gruel L. (2006), « Filles et garçons : Amphis, discothèques et lave-linge. Quelques clés pour comprendre les différences de réussite selon qu'on est fille ou garçon », OVE Infos, $\mathrm{n}^{\circ} 15$, mars.

Gruel L. (2009), « En guise de conclusion : quatre grands facteurs de différenciation ", in Gruel L., Galland O., Houzel G. (sous la direction de), Les étudiants en France. Histoire et sociologie d'une nouvelle jeunesse, Rennes, PUR, novembre pp. 389-397.

Lahire B. (1997), Les manières d'étudier, La Documentation française, Paris, Collection «Les Cahiers de l'OVE $», n^{\circ} 2$.

Merle P. (2000), " Le concept de démocratisation d'une institution scolaire : une typologie et sa mise à l'épreuve », Population, vol. 55, $n^{\circ}$ 1, pp. 15-50.
Millet M. (2003), Les étudiants et le travail universitaire, Lyon, Presses universitaires de Lyon.

Millet M., Thin D. (2007), " Scolarités singulières et déterminants sociologiques ", Revue française de pédagogie, $\mathrm{n}^{\circ}$ 161, décembre, pp. 41-51.

Musselin C. (2001), La longue marche des universités françaises, Paris, PUF.

Passeron J.-C. (1982), « L'inflation des diplômes. Remarques sur l'usages de quelques concepts analogues en sociologie ", Revue française de sociologie, XXIII-IV, pp. 551-584.

Peugny C. (2009), Le déclassement, Grasset.

Rey O. (2009), « Quelques lignes de force du champ universitaire des années 1990 à aujourd'hui », in Gruel L., Galland O., Houzel G. (sous la direction de), Les étudiants en France. Histoire et sociologie d'une nouvelle jeunesse, Rennes, PUR, novembre, pp. 381-387.

Ringer F. (2003), « La segmentation des systèmes d'enseignement ", Actes de la recherche en sciences sociales, $\mathrm{n}^{\circ} 149$, pp. 6-20.

Schnitzer K. (2008) (Dir.), Eurostudent Report, Social and Economic Conditions of Student Life, Hanovre, Synopsis of indicators, HIS.

\section{Résumé}

\section{L'enseignement supérieur en France : un espace segmenté qui limite l'égalisation des chances}

Elise Verley et Sandra Zilonniz

Alors que la part des étudiants inscrits à l'université tend à diminuer, les filières sélectives, parce qu'elles offrent des formations "prestigieuses » et/ou professionnalisantes, exercent une attractivité croissante. À l'heure de la stabilisation du nombre d'élèves et d'étudiants, les concurrences entre établissements s'aiguisent et conduisent à considérer l'enseignement supérieur comme un espace segmenté, ce quel que soit l'angle d'analyse retenu: profils social et scolaire, projets d'études, pratiques studieuses, conditions d'études et perspectives professionnelles. L'enquête "Conditions de vie " de l'Observatoire national de la vie étudiante permet de comparer jugements et anticipations des étudiants en premier cycle dans les différents segments de l'enseignement supérieur : universitaire, professionnalisant, sélectif.

Mots clés :

Accès à l'enseignement supérieur, enquête, étudiant, inégalités, filière de formation Journal of Economic Literature: I 23 